\title{
In-line Laser Thermography for Crack Detection: A Numerical Approach
}

\author{
Nithin P V1, Sreedhar Unnikrishnakurup1, C V Krishnamurthy1, Mathias Zeigler2, Philippe Myrach2 and \\ Krishnan Balasubramaniam \\ Centre for Non-Destructive Evaluation, Mechanical Engineering Department, \\ IIT Madras, India \\ 2 BAM Federal Institute for Materials Research and Testing, Division 8.7, Unter den Eichen 87, 12205 Berlin, \\ Germany \\ nithinvengara@gmail.com
}

\begin{abstract}
The detection and characterization of cracks prior to damage is a technologically and economically highly significant task and is critical when it comes to safety-relevant structures. The evaluation of a component's structural health is closely related to the presence of surface cracks. One of the more promising techniques among NDT techniques is thermography. In the present work a local laser spot excitation for surface crack detection is simulated using the finite element method (FEM). The FEM model simulates the heat diffusion phenomena and the effect of the presence of known surface crack on a NiCr coated brass sample. The transient heat transfer in the material is affected by the crack position and size and enabled us to detect the discontinuity in the surface temperature distribution. The variation of this surface thermal discontinuity is studied. The developed FEM model shows a good correlation with the experimental measurements.
\end{abstract}

Keywords: In-line Monitoring, Laser Thermography, FEM, Elevated temperature, Surface cracks.

\section{Introduction}

The detection of the surface breaking cracks in engineering components is an important task to prevent structural failure. Especially in the case of wide- spread use of low ductility, high performance steel in lightweight construction, this is a subject of increasing interest, where surface breaking cracks are the most critical in nature cause in service material failure. The detection and characterization of cracks prior to damage is a technologically and economically highly significant task and is of utmost importance when it comes to safety-relevant structures. The evaluation of a components structural health is closely related to the presence of cracks. Using non-destructive testing, such potential flaws can be identified before the occurrence of serious damages or even might allow for a repair. There are many NDT techniques detailed tackling this problem, like for instance eddy current, ultrasound, dye penetration, magnetic particle, thermography, and computer tomography testing. One of the more promising techniques amongst those is thermography. This technique has the following advantages over other NDT techniques:

- it is not limited to electrically conducting or ferromagnetic materials (e.g., eddy current, magnetic particle testing),

- it does not need environmentally harmful substances (e.g., dye penetration, magnetic particle testing),

- it does not need contact or close access to the test specimen (e.g., ultrasound, eddy current testing),

- it does not require component disassembly (e.g., computed tomography),

- it allows for fast scan and image acquisition times (e.g., less than one second),

- it directly provides images and allows for a precise online documentation.

- In contrast to the dye penetration testing that is, beside magnetic particle testing, one of the industrial standard test methods for surface cracks, the thermographic approach offers the possibility to detect cracks that are covered by a painting or that even lie within the bulk of the test specimen (close to the surface).

Among the thermographic methods, flying spot laser spot thermography which currently in intense research activities has a promising capability in surface crack detection. This approach uses a laser, which is scanned over the surface. Changes in the heat conductivity then lead to changes in the thermal footprint, which is used for crack detection. This method was suggested for the first time in the late 1960s [1] On-going research during the last years has identified the laser-thermography as a powerful thermographic technique, which has already proven its capability for the detection of surface cracks and for the characterization of the geometry of artificial surface flaws in metallic samples. Local heating of the sample surface is realized by a laser that is scanned over the surface. Local changes in the heat conductivity introduced by cracks then lead to changes in the laser's thermal footprint which is detected by an infrared camera. The pros of laser thermography methods are high speed data collection, 2D imaging, 3D quantitative measurements, non-contact remote technique, can handle hostile environment and can be employed for measurement of defects, process and product 
parameters. And the main cons of this method are need line-of-sight for making measurements and thermography measurements needs emissivity corrections.

Now a day's the laser scanning thermography has been proposed as a promising method for crack detection[2][3]. The use of a moving laser beam as a heat source allows transmitting heat energy over a long distance, precisely controlling the intensity and position of the laser beam, and creating thermal wave propagation along a target surface, making it possible to detect surface cracks. Li et al [4] utilized a high- power pulse laser of $21 \mathrm{~W}$ as a heat source for surface-breaking crack detection in a metallic structure. Then Schlichting et al successfully detected a surface crack using a high-power continuous wave $(\mathrm{CW})$ laser of $5.2 \mathrm{~W}$. However, the exposure of the target structure to repeated high-power laser beam scan resulting surface ablation [5].

In this paper, We modeled a FEM model to simulate the heat diffusion phenomena and the effect of the presence of known surface crack on sample. The transient heat transfer in the material is affected by the crack position and size and enabled us to detect the discontinuity in the surface temperature distribution. The variation of this surface thermal discontinuity due to the presence of crack is studied. The developed FEM model shows a good correlation with the experimental measurements.

\section{2D Numerical modeling}

In this chapter, a two-dimensional model is described using COMSOL Multiphysics to investigate the heat transport and resulting temperature change on the surface of the sample which consist of substrate plates made of brass.and substrate is covered with a Ni/Cr layer of thicknesses $50 \mu \mathrm{m}$, which define the actual depth of the cracks. specimen parameters are brass plate is $2 \mathrm{~mm}$ in thickness and $50 \mathrm{~mm}$ in width. Crack depth is $50 \mu \mathrm{m}$ and width is $12 \mu \mathrm{m}$. there are four cracks are modeled and a $63 \mathrm{~W}$ laser beam is scanning over the surface at a speed of $30 \mathrm{~mm} / \mathrm{s}$.

When laser beam on the surface a temperature gradient exists in the material, there is an energy transfer from the high-temperature region to the low-temperature region. The heat transfer rate due to conduction per unit area is proportional to the normal temperature gradient

$$
\frac{q}{A}=-k \frac{\partial T}{\partial x}
$$

Where $q$ is the heat transfer rate, $\frac{\partial T}{\partial x}$ is the temperature gradient in the direction of heat flow, and the constant $k$ is the thermal conductivity of the material $(\mathrm{W} / \mathrm{m}$. K). The minus sign is inserted to indicate that heat flows from a higher temperature value to a lower temperature value. Considering the energy balance in three dimension (energy in + heat generated within - energy out = change in internal energy), the heat conduction equation in $2 \mathrm{D}$ in a concise form, the equation can be of the form can be written as

$$
\nabla .(k \nabla T)+\dot{Q}=\rho C_{p} \frac{\partial T}{\partial t}
$$

Where $\dot{Q}$ is the heat generation, $\rho$ is the density of the material, and $C_{p}$ is the specific heat of the material. The initial value of the temperature is the room temperature $(T 0=293 \mathrm{~K})$ for all domains

Table1. Material properties

\begin{tabular}{|c|c|c|}
\hline Material property & Brass & NiCr alloy \\
\hline Thermal conductivity $k\left(\mathrm{Wm}^{-1} \mathrm{~K}^{-1}\right)$ & 120 & 13 \\
\hline Specific heat capacity $c\left(\mathrm{~J} \mathrm{~kg}^{-1} \mathrm{~K}^{-1}\right)$ & 375 & 480 \\
\hline Density $\rho\left(\mathrm{kgm}^{-3}\right)$ & 8530 & 8200 \\
\hline
\end{tabular}

A Gaussian laser beam is defined as the heat source and hence it can be written in mathematical form as described as bellow 


$$
Q_{\text {in }}=\left(1-R_{c}\right) \frac{q_{0}}{\pi r^{2}} \exp \left(\frac{-\left(x-x_{0}\right)^{2}}{r^{2}}\right)
$$

Where $Q_{\text {in }}\left[\mathrm{W} / \mathrm{m}^{2}\right]$ incident laser intensity, $R_{c}$ reflection co efficient, $q_{0}$ is the laser power and $r$ is the laser spot diameter and $x_{0}$ is spatial coordinate and is the function of laser scanning speed and time. Since the material surface is highly reflective high power laser can be use for scanning with optimal scanning speed.

\section{Crack geometry and meshing}

The model geometry is created in comsol multiphysics with sample parameter. The appropriate material properties are assigned for the geometry .and free triangular meshing applied for the enter geometry

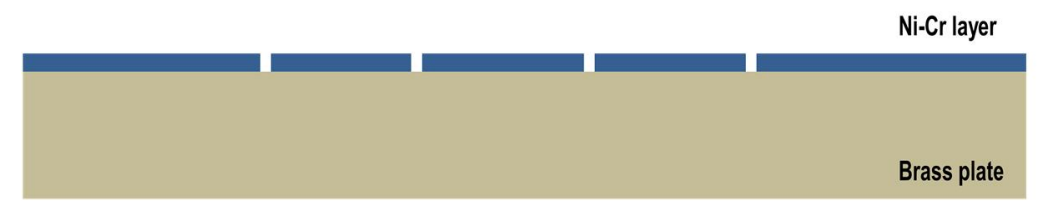

Fig.1. Model geometry

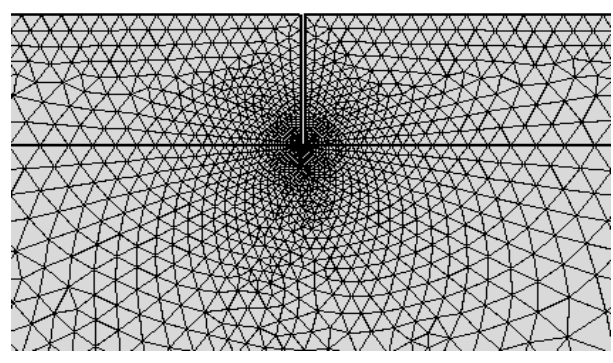

Fig.2. Mesh near a crack

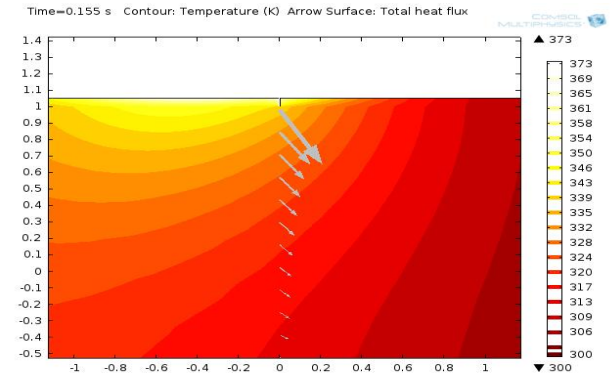

Fig.3. 2D FEM simulated heat distribution pattern across the thickness in the sample

In fig. 1 shows the geometry of the sample having four crack having the same width and depth. Fig 2 shows the meshing near a crack and the Fig. 3 is the simulated heat distribution pattern across the thickness of the sample and the presence of crack will disturb the heat distribution and this analogy can easily capture by a camera.

\section{Experimental Validation}

Fig.4. shows the schematic representation experimental setup, a high power diode laser and an optical scanner and an IR camera are the main components of the setup. For local heating we apply an experimental setup (see Fig. 1) consisting of a diode laser fibre coupled to an optical scanner(920 nm wavelength, $63 \mathrm{~W}$ continuous wave output power, 1.3 $\mathrm{mm}$ spot size, $20 \mathrm{~m} / \mathrm{s}$ maximum scanning speed) and a fixed InSb-based IR-camera $(640 \times 512$ pixel, $100 \mathrm{~Hz}$ frame rate, sensitive in the 3-5 $\mu \mathrm{m}$ wavelength range, $50 \mu \mathrm{m} /$ pixel spatial resolution). The sample surface is successively scanned in parallel lines in the same direction in a lateral distance according to the laser spot diameter. Using a dichroic mirror allows for operation in normal incidence avoiding image distortions and damages caused by the laser lighFig. 5 is the photo graph of the sample where cracks are introduced.

The influence of the actual crack geometry was studied on the specimen shown in figure 6 . These plates consist of substrate plates made of brass. The substrate is covered with a Ni/Cr layer of different thicknesses, which define the actual depth of the cracks. This set of specimens represents a particular difficult inspection problem for laser thermography, due to the high reflectance of its surfaces. Because of this high reflectance only a small amount of the applied energy is absorbed and can heat up the specimen. At the same time the low emissivity significantly reduces the amount of emitted IR radiation, which further handicaps the crack detection [6]. 


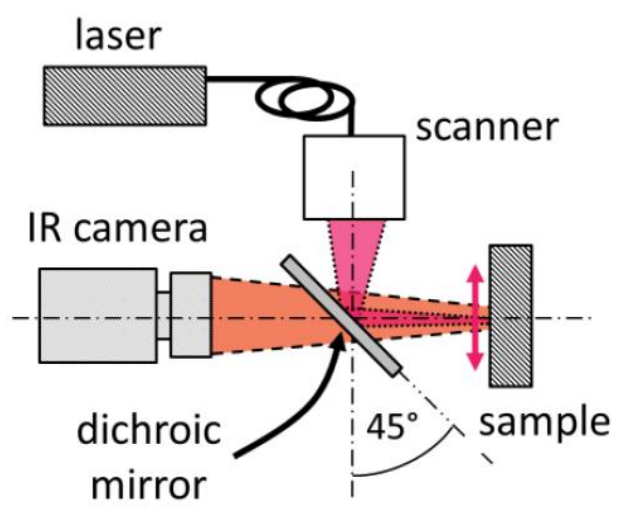

Fig.4. Schematic representation of the experimental set-up.

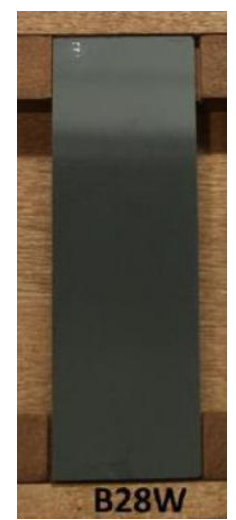

Fig.5. sample

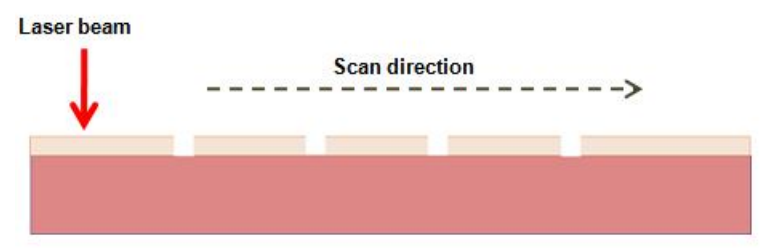

Fig.6. Schematic of laser scanning

The influence of the actual crack geometry was studied on the specimen shown in figure 6 . These plates consist of substrate plates made of brass. The substrate is covered with a Ni/Cr layer of different thicknesses, which define the actual depth of the cracks. This set of specimens represents a particular difficult inspection problem for laser thermography, due to the high reflectance of its surfaces. Because of this high reflectance only a small amount of the applied energy is absorbed and can heat up the specimen. At the same time the low emissivity significantly reduces the amount of emitted IR radiation, which further handicaps the crack detection.

The low absorption could be partly counteracted by the use of high laser power (63 W), small spot diameter and relatively low scanning speeds. Increasing the energy density by these measures provides a reasonable temperature rise on the surface to get crack indications.

\section{Results and discussion}

Laser line scan over the sample with $30 \mathrm{~mm} / \mathrm{s}$ scanning speed is carried out experimentally and the corresponding 2D FEM model developed. The Fig.7 and Fig.8 shows the thermal heat distribution due to the laser spot in radial direction. Fig.7 shows the experimental data obtained for a laser spot at a location where there is no crack and the second image shows the laser spot when it just crossed one of the cracks which results the heating of the crack and air present inside the crack so the presence of crack is highlighted in Fig.8. Most of the IR radiation is therefore reflected at the surface. When light enters a crack on the other hand it is reflected multiple times inside the crack and will deposit a larger amount of energy than at a single reflection, in a similar ways in a black-body cavity. A crack in a metal plate will absorb and emit more energy than the surroundings and will be visible as a hot-spot in thermo gram. 


\section{http://dx.doi.org/10.21611/qirt.2015.0089}

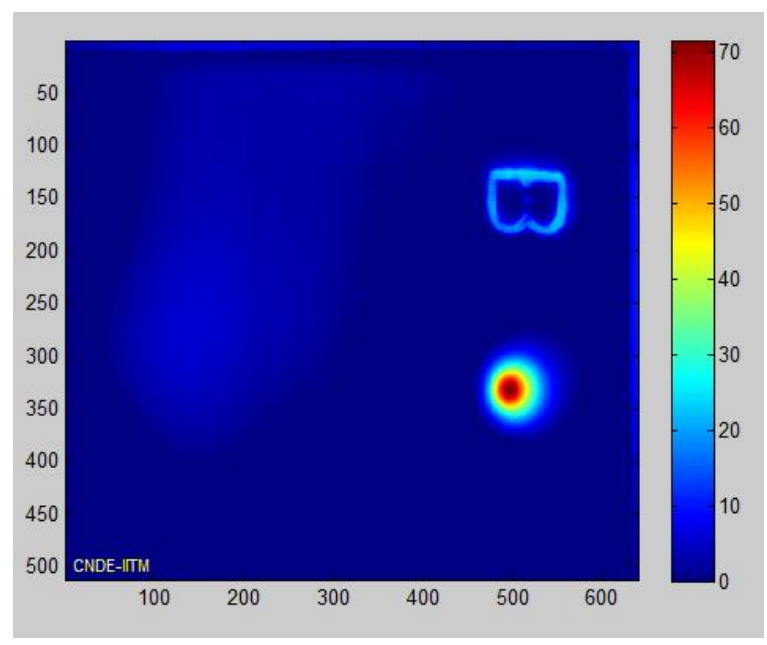

Fig.7. Laser spot before a crack

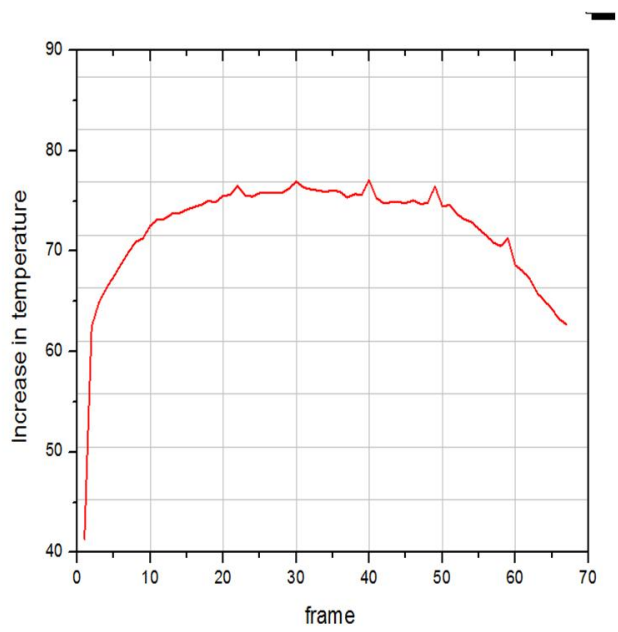

Fig.9. Timing graph for line of scanning- Experiment

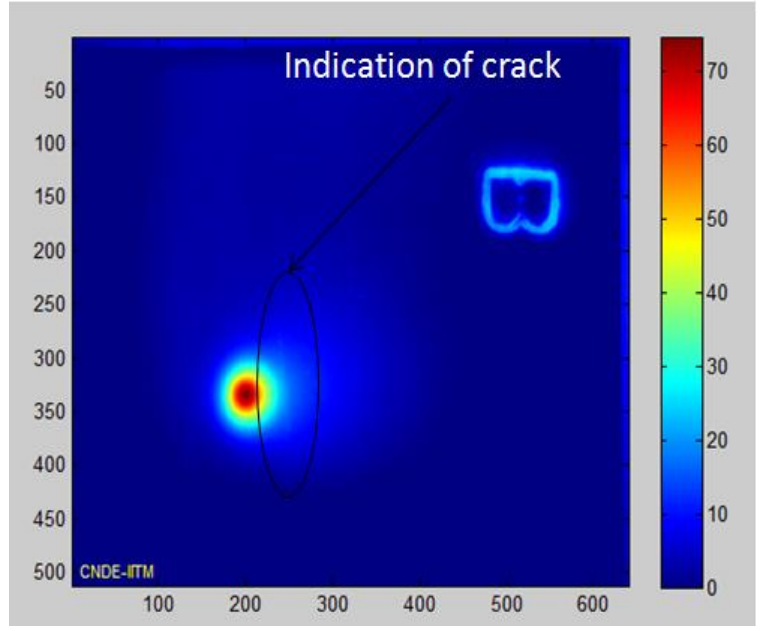

Fig.8. Laser spot just after a crack

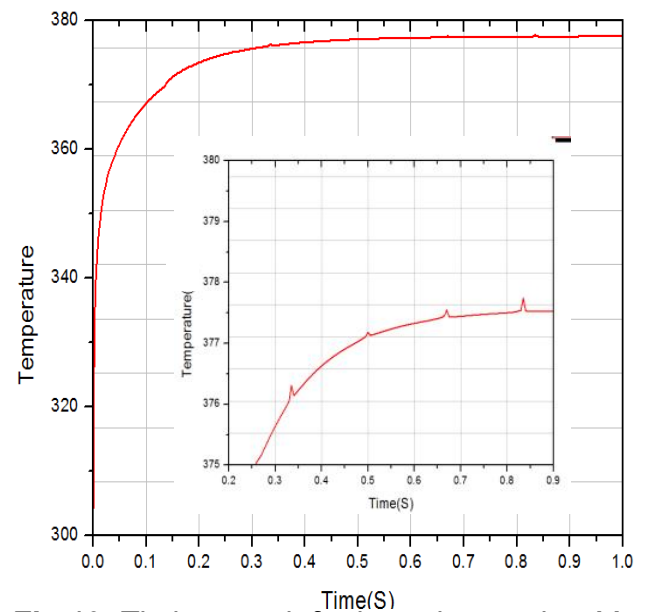

Fig.10. Timing graph for boundary probe- Modeling

Now we are considering a line profile through which laser beam is passing and drawn the timing graph for maximum temperature. The Fig.9 shows the timing graph for the line profile in experimental data, from the figure the presence of cracks easily identified as the increase in temperature. And Fig.10 is the same we obtained from modeling result, these results gives good correlation with the experimental data. There is a good correlation with material surface temperature rise due to laser scanning. Maximum temperature rise on the surface due to laser scan in experiment results is $77 \mathrm{~K}$ and $79 \mathrm{~K}$ by modeling. But the temperature rise due to the presence crack in experimental results is $1.8 \mathrm{~K}$ where as in modeling we are getting $0.1 \mathrm{~K}$ increase only, This is as expected because in the modeling we considered the temperature rise due to the heat blockage only we are not considered the temperature rise due to the optical trap due to the laser beam crack interaction

\section{Conclusions}

Simple 2D numerical scanning laser thermography using CW laser is modeled and validated with experimental results. Boundary probe analysis showed significant indications with the surface opening notch. The 2D modeling results gives good correlation with the experimental data there will be good correlation with material surface temperature rise due to laser scanning. 2Dmodel. But there is a variation in rise in temperature due to the presence of crack in experimental and modelling results. It is expected this variation because we are not considered the optical trap inside the crack which enhance the multiple reflections and the incident beam will completely absorb by crack surface It is possible further improving this model by including the crack-laser beam interaction physics, better correlations can be obtained. 


\section{Acknowledgements:}

This work is financially supported from Indo-German Science and Technological Centre (IGSTC) under the project entitled Advanced Manufacturing Process Monitoring using in-line LASer Thermography (AMPLAST).

\section{REFERENCES}

[1] E. J. Kubiak, "Infrared detection of fatigue cracks and other near-surface defects", Applied Optics, 7(9), 1743(1968).

[2] C. Gruss, D. Balageas, "Theoretical and experimental applications of the flying spot camera", Proc. QIRT 92 (Seminar Eurotherm No. 27), 19-24(1992)

[3] S. E. Burrows, A. Rashed, D. P. Almond, S. Dixon, "Combined laser spot imaging thermography and ultrasonic measurements for crack detection," NDT \&E Intern. (Special Issue: Thermographic NDT) 22 (2-3)(2007).

[4] T. Li, D. P. Almond, D. Andrew, S. Rees, B. Weekes, "Crack imaging by scanning pulsed laser spot thermography", NDT\&E Intern. 44, 216(2011)

[5] J. Schlichting, M. Ziegler, C. Maierhofer, M. Kreutzbruck, "Efficient data evaluation for thermographic crack detection", QIRT Journal, Short Communications 8(1), 119(2011).

[6] Philipp Myrach, Mathias Ziegler, Christiane Maierhofer, Marc Kreutzbruck "Influence of the Acquisition Parameters on the Performance of Laser-Thermography for Crack Detection in Metallic Components", proc. QNDE2013(2013) 